\title{
Signal-Timing Offset Compensation in Dense TDD OFDM-based Networks
}

\author{
Adrián Agustín, Sandra Lagén, Josep Vidal \\ Signal Processing and Communications Group (SPCOM), Universitat Politècnica de Catalunya, Barcelona, Spain
}

\begin{abstract}
Orthogonal Frequency Division Multiplexing (OFDM)based networks rely on time synchronization to obtain their best performance. Time synchronization with neighboring nodes can be satisfied by increasing the cyclic prefix (CP) length (at the expenses of spectral efficiency reduction) We show that by optimizing the transmit precompensation and receive post-compensation we can meet the time synchronization constraints and keep the $C P$ at its minimum value. This concept is applied to paired-bands Frequency Division Duplexing (FDD) systems which tend to show inefficient occupancy of the uplink (UL)-band due to the traffic asymmetry. We consider the possibility of deploying multiple Time Division Duplexing (TDD) small eNBs (SeNBs) in the unused UL spectrum. In this scenario, time synchronization with macro eNB (MeNB) and neighboring SeNBs becomes essential. Two algorithms are proposed in order to ensure orthogonality of OFDM transmissions network-wide.
\end{abstract}

Keywords—Cyclic Prefix, OFDM, synchronization

\section{INTRODUCTION}

In recent years a tremendous research effort has been devoted to improve the spectral efficiency of wireless systems as a result of the increased traffic demands generated by mobile users. At the same time, paired spectrum bands have been licensed and deployed while traffic demand has shown clear asymmetries between UL and DL [1].

One of the envisioned solutions to combat the issue is to densify the cellular network with small cells (SeNBs) that provide short range coverage, [2], so that the spectrum can be spatially reused. Another research direction advocates for efficient spectrum usage by means of flexible duplexing [3]. While cellular TDD-based networks can adapt the DL/UL frame pattern as a function of the traffic, FDD-based networks should be able to adapt the bandwidth devoted for DL and UL transmissions. However, FDD-based networks with a preassigned paired spectrum tend to have a large amount of unused resources in the FDD-UL band, because of the traffic asymmetry. Current 3GPP LTE-A standard and regulation hamper the use of those unused resources for DL [4][5].

From the technical point of view, such resources could be efficiently exploited if multiple TDD SeNBs operated in the unused carriers (Figure 1). This new scenario with FDD-UL transmissions coexisting with TDD transmissions in the same band demands revisiting the conventional methods to tackle network synchronization. This aspect is also relevant in 5G systems (most likely based on OFDM), where the time frame pattern is being redesigned, including the CP length. Scenarios with a large number of SeNBs (ultra dense networks) are envisioned, and adequate time synchronization is desired to avoid asynchronous interference.

The most common way to do it is to distribute a common time reference over the network, which is used by the

This work has been supported by Huawei Technologies Co., Ltd. Also by the "Ministerio de Economía y Competitividad" of the Spanish Government and ERDF funds (TEC2013-41315-R DISNET), and from project 5G\&B RUNNER-UPC (TEC2016-77148-C2-1-R (AEI/FEDER, UE)
MeNBs/SeNBs to initiate DL transmissions, [6]. Notice that CP is usually considered to deal with the multipath impairment but it also plays a crucial role in avoiding inter-user interference in the UL of OFDMA systems. For the UL, all transmissions from UEs are pre-compensated (time-advance procedure) with the objective that signals arrive at the MeNB/SeNB within certain time interval, i.e. the cyclic prefix (CP) in OFDMA-based systems [7]. Currently, the UL pre-compensation is calculated just considering the serving MeNB, but with the introduction of SeNBs this aspect should be rethought as it is pointed in [8], where a SeNB is deployed in a macro cell. In such a case, UEs associated to SeNB have to advance their UL transmissions so as to be aligned with the UL frame boundary at MeNB and SeNB. Furthermore, SeNB should adjust its UL frame boundary taking into account the MeNB one. This is required to keep the orthogonality among the OFDM carriers and avoid performance degradation due to interference [9][10]. Figure 1 shows two TDD SeNBs coexisting in the FDD UL band of a macro cell. The signals coming from $\mathrm{SeNB}_{2}$ and MUE arrive at MeNB in the CP time, but not at $\mathrm{SeNB}_{1}$, and losing their orthogonality because there are different symbols from the same source in the receive window.

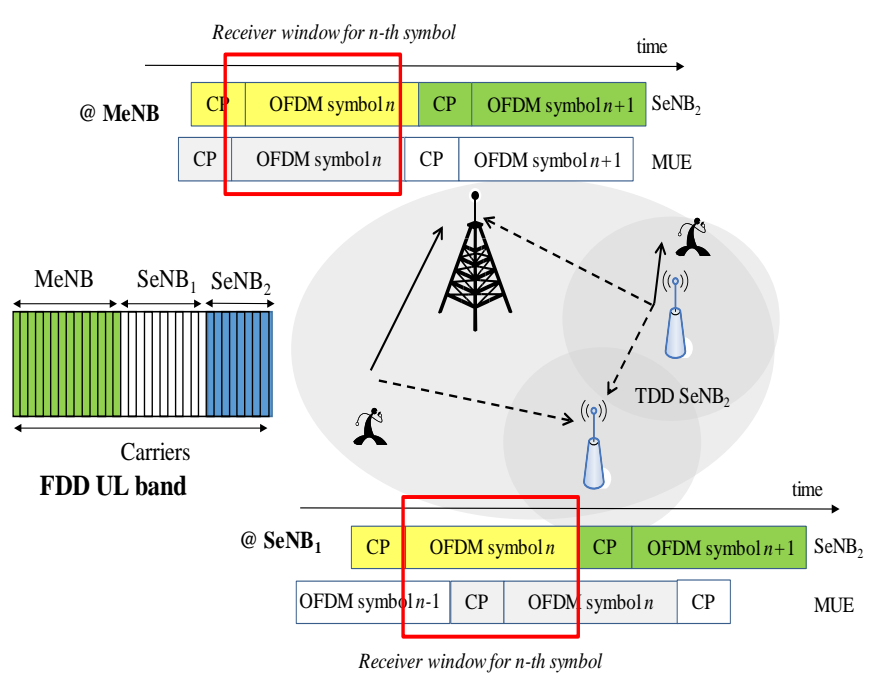

Fig. 1. Spectrum coexistence of FDD-UL MeNB and TDD SeNBs. SeNB 1 and MeNB are receiving. MUE and $\mathrm{SeNB}_{2}$ are transmiting UL and DL signals respectively. Both signals arrive within the CP time interval at the MeNB but not at the $\mathrm{SeNB}_{1}$, that receives signal from two different symbols $(n-1, n)$.

In this work we address the problem of time synchronization for maintaining the orthogonality among carriers in OFDM when multiple TDD SeNBs operate in the FDD-UL band and 
coexist with MeNBs. This scenario, see Figure 1, has not been addressed in previous works [11][12][13]. The challenge faced is the removal of the asynchronous interference among SeNBs and MeNBs, i.e. multipoint-to-multipoint scenario with different signal-timing offsets (STO) between nodes. In [14] a similar scenario is considered and an algorithm is proposed to adjust the pre-compensation for coordinated multipoint-tomultiuser systems. The present work deals with additional constraints originated by the neighboring TDD SeNBs coexisting with the FDD-UL MeNB. We will elucidate the benefits of optimizing transmit pre-compensation and receive post-compensation in multi-terminal OFDM-based networks, as well as selecting the shortest CP length that allows maintaining the orthogonality of the carriers in reception. Our main contributions are:

- Two algorithms are proposed to design the transmitter pre-compensation, receiver post-compensation, and the minimum CP length at SeNBs. Both are obtained by formulating and solving a convex optimization problem.

- A new user association and admission algorithm is proposed that takes into account the synchronization constraints.

\section{SYSTEM MODEL}

We assume a coverage area where $N_{M e N B}$ MeNBs are operating in FDD, defined by the set $\mathcal{M}=\left\{1, . ., N_{\text {MeNB }}\right\}$, and providing service to $N_{U E s}$ UEs, $\mathcal{U}=\left\{1, . ., N_{U E s}\right\}$ uniformly distributed over the area. Further, up to $N_{S e N B}$ TDD SeNBs, $\mathcal{S}=\left\{1, . ., N_{\text {SeNB }}\right\}$, are deployed in the FDD-UL band with the objective of seizing the resources unused by conventional FDD macro cells in the UL. Thus, MeNB and SeNBs access the channel under frequency division multiplexing, with a common $\mathrm{CP}$. The maximum channel dispersion at MeNBs and SeNBs is assumed to be the same, $\tau_{\max }$. Frequency offsets between terminals are not considered.

The STOs, measured in samples, between different nodes (i.e. $A$ and $B$ ) in the network depend on the distance between them $\left(d^{A-B}\right)$, the sampling frequency of the OFDM system ( $\left.T_{s}=1 / 2048 / 15 \times 10^{3}\right)$ and the speed of light $\left(3 \times 10^{8} \mathrm{~m} / \mathrm{s}\right)$ :

$$
t^{A-B}=d^{A-B} / 3 \times 10^{8} \times T_{s}
$$

We assume that these values are already known by the system and defined as,

$$
\begin{cases}t_{n, m}^{U E-M e N B}, t_{n, k}^{U E-S e N B} & n \in \mathcal{U}, m \in \mathcal{M}, k \in \mathcal{S} \\ t_{l, k}^{S e N B-S e N B}, t_{l, m}^{S e N B-M e N B} & k, l \in \mathcal{S}, k \neq l, m \in \mathcal{M}\end{cases}
$$

SeNBs are allowed to select the TDD duplexing frame pattern independently, i.e. without considering the decisions of neighboring SeNBs. In this regard, the system should be designed in a worst-case scenario where all SeNBs have to be synchronized among them and towards the FDD MeNBs, which are operating in the FDD-UL band. Similarly, synchronization is needed between SeNBs and the UEs associated to the MeNBs (MUEs).

Adjustment of the synchronization is done through transmission timing (transmitter pre-compensation) and the receiver sampling time (receiver post-compensation), so that all signals arrive within the CP time interval at a particular receiver. The parameters allowing synchronization are the following:

a) transmitter pre-compensation (time advance) at UE and SeNBs:

$$
\bar{x}_{n} \quad n \in \mathcal{U} \quad x_{l} \quad l \in \mathcal{S}
$$

b) receiver post-compensation (sampling time offset) at SeNBs and MeNBs:

$$
y_{l} \quad l \in \mathcal{S} \quad \bar{y}_{m} \quad m \in \mathcal{M}
$$

c) Cyclic prefix length at SeNBs and MeNBs: $C P$

Figure 2 illustrates the different signal arrivals at the MeNB and $\mathrm{SeNB}_{1}$ in the scenario presented in Figure 1 . It is assumed that the receive time reference at $\mathrm{SNB}_{1}$ and MeNB is the same and it is denoted by $\varepsilon_{0}$. The signals transmitted by MUE (assuming pre-compensation $\bar{x}_{1}$ ) and $\mathrm{SeNB}_{2}$ (assuming pre-compensation $\left.x_{2}\right)$ arrive at MeNB and SeNB as depicted in Figure 2-top and Figure 2-bottom, respectively. The receivers at MeNB and SeNB $_{1}$ apply receiver post-compensation $\left(\bar{y}_{1}\right)$ and $\left(y_{1}\right)$, respectively. Blue arrows in Figure 2 denote the time arrival difference between the received signals from MUE and $\mathrm{SeNB}_{2}$ with respect the new adjusted reference at MeNB and SeNB ${ }_{1}$.
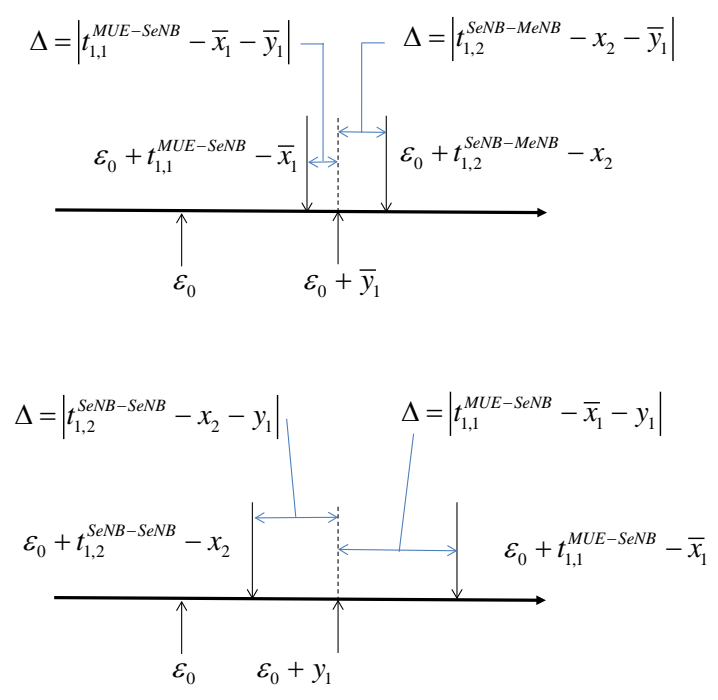

Fig. 2. Received signals at MeNB (top) and $\mathrm{SeNB}_{1}$ (bottom) from MUE and SeNB2. MeNB and SeNB apply post-compensation $\bar{y}_{1}, y_{1}$, respectively. Blue arrows indicate the time difference between the adjusted receive reference and the received signals.

In order to maintain the orthogonality of carriers, all signals must reach the receiver within the $\mathrm{CP}$ time interval (taking into account the multipath delay spread, $\tau_{\max }$ ). The example shown in Figure 2 can be expressed mathematically as,

$$
\begin{cases}\left|t_{2,1}^{S e N B-S e N B}-x_{2}-y_{1}\right| \leq \kappa, & \left|t_{1,1}^{U E-S e N B}-\bar{x}_{1}-y_{1}\right| \leq \kappa \\ \left|t_{2,1}^{S e N B-M e N B}-x_{2}-\bar{y}_{1}\right| \leq \kappa, & \left|t_{1,1}^{U E-M e N B}-\bar{x}_{1}-\bar{y}_{1}\right| \leq \kappa\end{cases}
$$

with $\kappa=\frac{1}{2}\left(C P-\tau_{\max }\right)$ 


\section{Signal TIMING Optimization}

The design of the variables described in section II that meet the time synchronization in the network can be obtained as the solution of the optimization problem:

$$
\begin{aligned}
&(\mathrm{P} 1)_{C P,\left\{x_{l}\right\},\left\{y_{l}\right\},\left\{\bar{x}_{n}\right\},\left\{\bar{y}_{m}\right\}} \quad f(C P) \\
& \text { s.t. } \quad\left(t_{l, k}^{\text {SeNB-SeNB }}-x_{l}-y_{k}\right)^{2} \leq\left(\frac{1}{2}\left(C P-\tau_{\max }\right)\right)^{2} \\
&\left(t_{l, m}^{\text {SeNB-MeNB }}-x_{l}-\bar{y}_{m}\right)^{2} \leq\left(\frac{1}{2}\left(C P-\tau_{\max }\right)\right)^{2} \\
& x_{l}-x_{l}^{\max } \leq 0, \quad-x_{l} \leq 0 \\
& C P-C P_{\max } \leq 0, \quad-C P \leq 0 \\
&\left(t_{n, k}^{U E-S e N B}-\bar{x}_{n}-y_{k}\right)^{2} \leq\left(\frac{1}{2}\left(C P-\tau_{\max }\right)\right)^{2} \\
&\left(t_{n, m}^{U E-M e N B}-\bar{x}_{n}-\bar{y}_{m}\right)^{2} \leq\left(\frac{1}{2}\left(C P-\tau_{\max }\right)\right)^{2} \\
& \forall l \neq k, \quad l, k \in \mathcal{S}, m \in \mathcal{M}, n \in \mathcal{U}
\end{aligned}
$$

where the objective is minimizing the CP length, where $f($.$) is$ an increasing function, variables $t_{n, m}^{U E-M e N B}, t_{n, k}^{U E-S e N B}$, $t_{l, k}^{S e N B-S e N B}, t_{l, m}^{S e N B-M e N B}$ are defined in (2), $\tau_{\max }$ is the maximum delay spread of the channel, $x_{l}, y_{l}$ are the transmit precompensation and receive post-compensation of the $l$-th SeNB when is operating in DL or UL, respectively, $\bar{x}_{n}$ denotes the transmit pre-compensation of the $n$-th UE and $\bar{y}_{m}$ the receive post-compensation at the $m$-th MeNB. Notice that the first two constraints in (6) impose that the signals transmitted by SeNB are synchronized with other SeNBs and MeNBs, while the last two constraints in (6) define that UEs are synchronous with MeNBs and SeNBs in UL.

This problem is not convex because of the presence of CP in the first and last constraints in (6). In this regard we propose two alternative approaches. The first in section II.A proposes a convex re-formulation that allows obtaining a semi-closed form solution, while the second in section II.B takes into account that the CP, post-compensation of MeNB and pre-compensation of UEs are fixed parameters, while only SeNB pre-compensation and post-compensation can be tuned to meet time synchronization. Finally, section II.C describes strategies in case the problem is not feasible.

\section{A. Convexifying $P 1$}

With the objective of formulating a convex problem we concentrate on minimizing the difference between the $\mathrm{CP}$ and the channel dispersion, i.e.

$$
Z=\frac{1}{4}\left(C P-\tau_{\max }\right)^{2}, \quad Z_{\max }=\frac{1}{4}\left(C P_{\max }-\tau_{\max }\right)^{2}
$$

Now the problem is recast as in equation (8), where $\left\{\lambda_{l, k}\right\},\left\{\omega_{l, m}\right\}$ denote the Lagrange multipliers associated to the constraints where the $l$-th SeNB is synchronized with the $k$-th SeNB and $m$-th MeNB, while $\left\{\psi_{n, k}\right\},\left\{\pi_{n, m}\right\}$ are the Lagrange multipliers of the synchronization constraints over the $n$-th UE towards the $k$-th SeNB and $m$-th MeNB.

$$
\begin{gathered}
\text { (P2) } \underset{Z,\{x l\},\{y l\},\left\{\bar{x}_{n}\right\},\left\{\bar{y}_{m}\right\}}{\operatorname{minimize}} Z^{2} \\
\text { s.t. }\left(\lambda_{l, k}\right):\left(t_{l, k}^{\text {SeNB-SeNB }}-x_{l}-y_{k}\right)^{2}-Z \leq 0 \\
\left(\omega_{l, m}\right):\left(t_{l, m}^{S e N B-M e N B}-x_{l}-\bar{y}_{m}\right)^{2}-Z \leq 0 \\
x_{l}-x_{l}^{\max } \leq 0, \quad-x_{l} \leq 0 \\
Z-Z_{\max } \leq 0, \quad-Z \leq 0 \\
\left(\psi_{n, k}\right):\left(t_{n, k}^{U E-S e N B}-\bar{x}_{n}-y_{k}\right)^{2}-Z \leq 0 \\
\left(\pi_{n, m}\right):\left(t_{n, m}^{U E-M e N B}-\bar{x}_{n}-\bar{y}_{m}\right)^{2}-Z \leq 0 \\
\forall l \neq k, \quad l, k \in \mathcal{S}, m \in \mathcal{M}, n \in \mathcal{U}
\end{gathered}
$$

Since now the problem in (8) is convex it can be solved using the dual method [15], whereby we obtain a semi-closed solution for the optimization variables as a function of the Lagrange multipliers or dual variables,

$$
\begin{gathered}
X_{l}=\frac{\sum_{k, k \neq l} \lambda_{l, k}\left(t_{l, k}^{S e N B-S e N B}-y_{k}\right)+\sum_{m} \omega_{l, m}\left(t_{l, m}^{S e N B-M e N B}-\bar{y}_{m}\right)}{\sum_{k, k \neq l} \lambda_{l, k}+\sum_{m} \omega_{l, m}} \\
\bar{X}_{n}=\frac{\sum_{k} \psi_{n, k}\left(t_{n, k}^{U E-S e N B}-y_{k}\right)+\sum_{m} \pi_{n, m}\left(t_{n, m}^{U E-M e N B}-\bar{y}_{m}\right)}{\sum_{k} \psi_{n, k}+\sum_{m} \pi_{n, m}} \\
y_{k}=\frac{\sum_{l, l \neq k} \lambda_{l, k}\left(t_{l, k}^{S e N B-S e N B}-x_{l}\right)+\sum_{n} \psi_{n, k}\left(t_{n, k}^{U E-S e N B}-\bar{x}_{n}\right)}{\sum_{l, l \neq k} \lambda_{l, k}+\sum_{n} \psi_{n, k}} \\
\bar{y}_{m}=\frac{\sum_{l} \omega_{l, m}\left(t_{l, m}^{S e N B-M e N B}-x_{l}\right)+\sum_{n} \pi_{n, m}\left(t_{n, k}^{U E-M e N B}-\bar{x}_{n}\right)}{\sum_{l} \omega_{l, m}+\sum_{n} \pi_{n, m}} \\
Z=\left.\frac{1}{2}\left(\sum_{l, k} \lambda_{l, k}+\sum_{l, m} \omega_{l, m}+\sum_{n, k} \psi_{n, k}+\sum_{n, m} \pi_{n, m}\right)\right|_{0} ^{Z_{\max }}
\end{gathered}
$$

The optimal primal variables can be obtained after using an iterative algorithm based on the sub-gradient approach [16] that consists on updating the Lagrange multipliers, decreasing or increasing them depending on whether the associated constraint is satisfied or not.

\section{B. Select timing given CP and association}

In the following we propose an algorithm that designs the system parameters for a scenario where all UEs are already associated to MeNBs and the CP in the network is fixed. Thus, we only have to elucidate the adequate timing of SeNBs that enable them to operate in the unused carriers of the MeNB FDD-UL band. We propose to set the active SeNBs and define its pre-compensation and post-compensation parameters as the 
solution of the following convex problem, assuming that the given $\mathrm{CP}$ (i.e. $C P_{\max }$ ) defines $Z_{\max }$ according to (7),

$$
\begin{aligned}
& \text { (P3) } \underset{\left\{x_{l}\right\},\left\{y_{l}\right\}}{\operatorname{minimize}}-\sum_{l} \log \left(x_{l}\right) \\
& \text { s.t. }\left(\lambda_{l, k}\right):\left(t_{l, k}^{\text {SeNB-SeNB }}-x_{l}-y_{k}\right)^{2}-Z_{\max } \leq 0, \quad \forall l \neq k \\
& \left(\omega_{l, m}\right):\left(t_{l, m}^{S e N B-M e N B}-x_{l}-\bar{y}_{m}\right)^{2}-Z_{\max } \leq 0, \\
& \left(\psi_{n, k}\right):\left(t_{n, k}^{U E-S e N B}-\bar{x}_{n}-y_{k}\right)^{2}-Z_{\max } \leq 0, \\
& \quad x_{l}-x_{l}^{\max } \leq 0, \quad-x_{l} \leq-1, \\
& \forall l, k \in \mathcal{S}, m \in \mathcal{M}, n \in \mathcal{U},
\end{aligned}
$$

We can obtain a semi-closed solution based on the dual variables, similarly to what was done in the previous section,

$$
\begin{gathered}
x_{l}=\frac{1}{2 \beta_{l}}\left(\theta_{l}+\sqrt{\theta_{l}^{2}+2 \beta_{l}}\right) \\
\theta_{l}=\sum_{k, k \neq l} \lambda_{l, k}\left(t_{l, k}^{S e N B-S e N B}-y_{k}\right)+\sum_{m} \omega_{l, m}\left(t_{l, m}^{S e N B-M e N B}-\bar{y}_{m}\right) \\
\beta_{l}=\sum_{k, k \neq l} \lambda_{l, k}+\sum_{m} \omega_{l, m} \\
\sum_{k}=\frac{\sum_{l, l \neq k} \lambda_{l, k}\left(t_{l, k}^{S e N B-S e N B}-x_{l}\right)+\sum_{n} \psi_{n, k}\left(t_{l, k}^{U E-S e N B}-\bar{x}_{n}\right)}{\sum_{l, l \neq k} \lambda_{l, k}+\sum_{n} \psi_{n, k}}
\end{gathered}
$$

\section{Feasibility}

It is important to remark that the problems presented in sections II.A and II.B might be infeasible (some of the constraints cannot be satisfied). Nevertheless, the solutions obtained above allow detecting if infeasibility is generated by a specific SeNB, and thus remove it accordingly. When a convex problem is infeasible, the Lagrangian grows unbounded with respect the dual variables, [15]. In such a case, we should not allow the SeNB having a larger contribution to the Lagrangian to transmit, and remove it from the problem accordingly. A similar approach was considered in [17].

\section{ASSOCIATION CRITERIA FOR NEW UES}

In the previous section we have presented two algorithms that successfully configure a set of active SeNBs while guaranteeing all synchronization requirements with neighboring MeNBs, other SeNBs and existing MUEs. The optimized variables (SeNB post- and pre-compensation and CP) are then kept fixed, and SeNBs can start to accept new UEs (SUEs). In the following we present two association criteria for new UEs coming up in the system. The pros- and cons- of the algorithms are evaluated in section $\mathrm{V}$.

\section{A. Greedy}

Each UE tries to be associated with the best SeNB or MeNB, i.e. the one that is received with the largest signal-to-noise ratio (SNR) along with a cell range expansion (CRE), [18] and tries to pre-compensate its transmission timing. It will be referenced as the $k^{*}$-th access point. As a criterion, we consider to find the minimum pre-compensation that satisfy the synchronization constraint with the selected access point. At the $n$-th UE, this can be obtained as,

$$
\begin{aligned}
& \underset{\left\{\bar{x}_{n}\right\}}{\operatorname{minimize}} \bar{X}_{n} \\
& \text { s.t. }\left(t_{n, k^{*}}^{U E-t x}-\bar{X}_{n}-w_{k^{*}}\right)^{2}-Z \leq 0
\end{aligned}
$$

with

$$
t_{n, k^{*}}^{U E-t x}=\left\{\begin{array}{ll}
t_{n, k^{*}}^{U E-S e N B} & \text { if } M e N B \\
t_{n, k^{*}}^{U E-M e N B} & \text { if SeNB }
\end{array}, w_{k^{*}}= \begin{cases}\bar{y}_{k^{*}} & \text { if } M e N B \\
y_{k^{*}} & \text { if } \operatorname{SeNB}\end{cases}\right.
$$

\section{B. Fair}

User association and UE transmitter pre-compensation are designed jointly, considering time synchronization constraints with all neighboring MeNBs and SeNBs, and selecting the best access point that satisfy all the constraints, in this regard we propose that each UE selects the minimum transmit precompensation according to,

$$
\begin{aligned}
& \underset{\left\{\bar{x}_{n}\right\}}{\operatorname{minimize}} \bar{x}_{n} \\
& \text { s.t. }\left(t_{n, k}^{U E-S e N B}-\bar{x}_{n}-y_{k}\right)^{2}-Z \leq 0 \quad \forall k \in \mathcal{S} \\
& \left(t_{n, m}^{U E-M e N B}-\bar{x}_{n}-\bar{y}_{m}\right)^{2}-Z \leq 0 \quad \forall m \in \mathcal{M}
\end{aligned}
$$

\section{RESUlTS}

The results are organized into specific questions. We assume 3 MeNBs at inter-site distance of $500 \mathrm{~m}$ and the maximum channel dispersion $\tau_{\max }=1 \mu \mathrm{s}$. The number of SeNBs is $N_{S e N B}=\{3,6,12,18\}$.

\section{A. Impact of greedy vs fair MUEs association}

In a scenario without SeNBs, we would like to assess the impact of the greedy or fair association criteria on the system performance. Figure 3 depicts the percentage-wise of UEs that are not synchronized to all MeNBs as a function of the CP length. Clearly, the fair criterion allows having more synchronized users with a significantly lower CP.

\section{B. Synchronization of SeNBs}

In case we could optimize all system parameters (transmit pre-compensations and receive post-compensations) for UEs, MUEs and SeNBs, the algorithm proposed in section III.A, problem P2 applies. In our scenario it provides a CP length of 112 (for different number of deployed SeNBs), see Figure 4. Additionally, Figure 4 depicts the case when transmit precompensation of SeNBs is adjusted to be synchronized either with best MeNB (greedy, see section IV.A) or with neighboring MeNBs (fair, see section IV.B), but receive postcompensations at MeNB and SeNBs are not optimized. Figure 4 illustrates the percentage of deployments that SeNBs satisfy the synchronization constraints with other SeNBs, without taking into account MUE synchronization constraints. We can observe that the SeNBs can be deployed with a smaller CP length in case of using the fair criterion. 


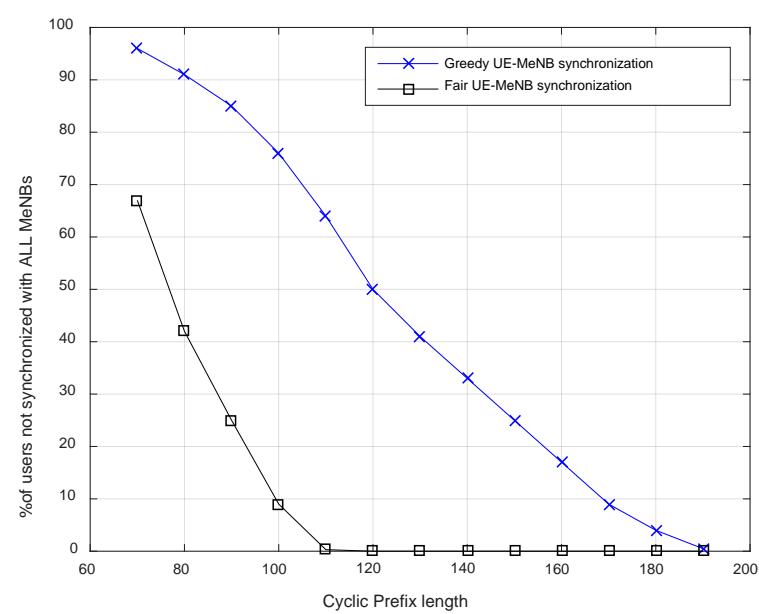

Fig. 3. Percentage-wise of UEs not synchronized to all neighboring MeNBs as a function of the CP length $\left(\mathrm{T}_{\mathrm{s}}\right)$.

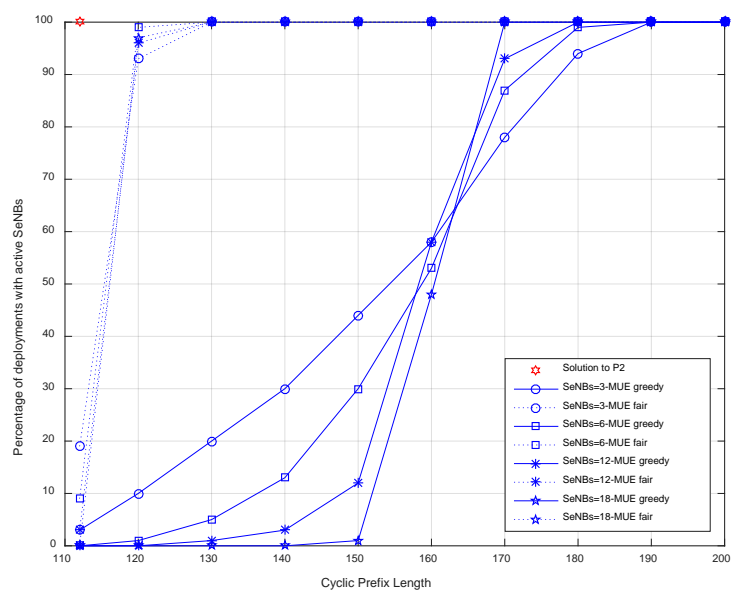

Fig. 4. Percentage-wise of deployments that SeNB satisfy synchronization constraints with other SeNBs as a function of the CP length $\left(T_{s}\right)$ in samples.

\section{Benefits of tunning the post-compensation of SeNBs}

Although the previous results are very promising, we should consider that in our scenario MUEs' transmissions in the UL coexist with SeNBs. In case the MUEs' transmit precompensation is fixed before-hand, the SeNB transmissions should be designed in such a way that transmissions from MUEs are received within the CP time interval, thus receive post-compensation is fundamental. Figure 5 depicts the percentage-wise of deployments when all synchronization constraints are satisfied, comparing solutions to problems P2 and P3 and the static solution where only transmit precompensations of SeNBs are adjusted according to a fair criterion. This latter approach can only increase the CP length so as to allow that signals from MUEs arrive synchronously to all SeNBs. On the other hand, the proposed problem P3 is able to deal with smaller CP length because it designs the receive post-compensation and additionally it can switch off those SeNBs that cannot cope with the signals from MUEs. In this regard, Figure 6 depicts the average number of SeNB that remain switched on, using the solution to problem $\mathrm{P} 3$, as a function of the CP length.

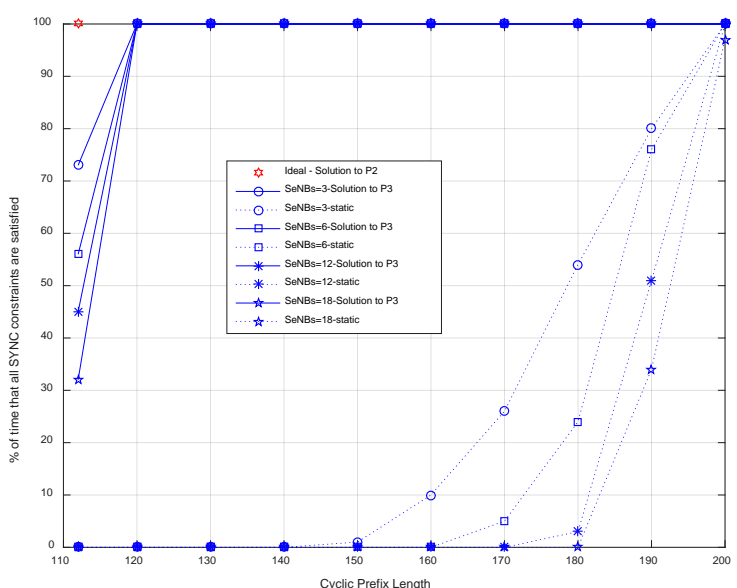

Fig 5. Percentage-wise of deployments that all synchronization constraints are satisfied comparing solutions to problems P2 and P3 and the static approach described in section V.B

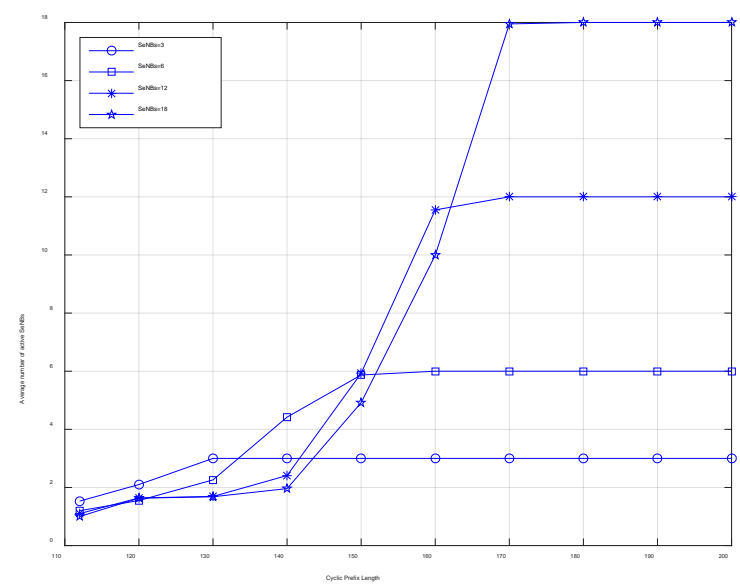

Fig. 6. Average number of active SeNBs using problem P3.

\section{Offloading the MBS}

Once the SeNBs have been configured to work using the solution of P3, they can start accepting UEs and offloading the macro cell. Nevertheless, the synchronization constraints should be considered when a SeNB accepts a SUE, see section $\mathrm{IV}$, otherwise asynchronous interference originated by the new SUEs will come up in the system. In this regard, we have considered one set of MUEs in order to optimize the system parameters of SeNBs (post- and pre-compensation) and afterwards we have simulated a new set of UEs that have to decide if becoming associated to MeNBs or to active SeNBs. Figure 7 shows that there is a large percentage of outages (defined here as at least one UE does not satisfy any synchronization constraint). Notice that when we increase the CP length, problem P3 adjusts the actual number of active SeNBs, see Figure 6. This is the reason why the outage increases for certain values of the $\mathrm{CP}$, while it decreases with the larger ones, once all SeNB are always working. 


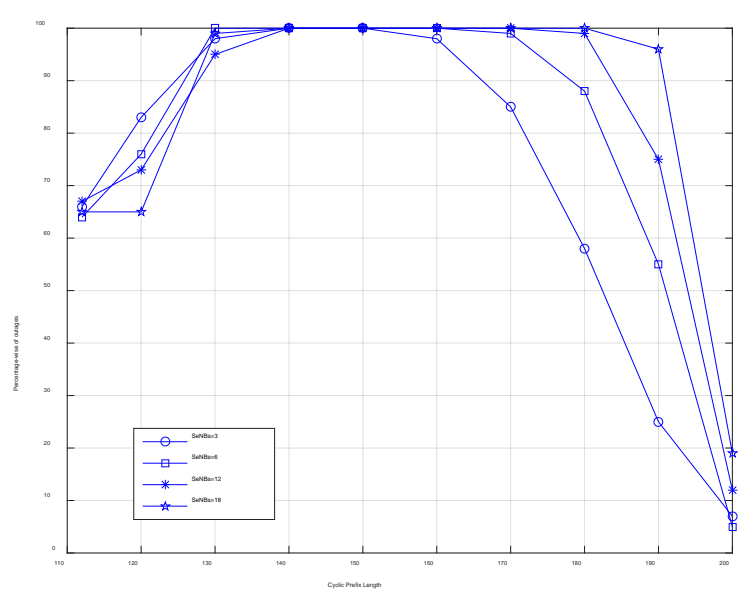

Fig. 7. Percentage-wise of at least one UE does not satisfy synchronization constraints with SeNBs and MeNB, once it has been associated to a SeNB.

Finally, Figure 8 describes the percentage of offloaded users in the network thanks to the deployment of multiple SeNBs using the solution to $\mathrm{P} 3$ and considering that the association also takes into account the fair criterion explained in section IV.B. We can observe that a large percentage of users can be offloaded satisfactorily to the TDD SeNBs: up to 50\% when the maximum SeNBs are 18 and 15\% when there 3 SeNBs.

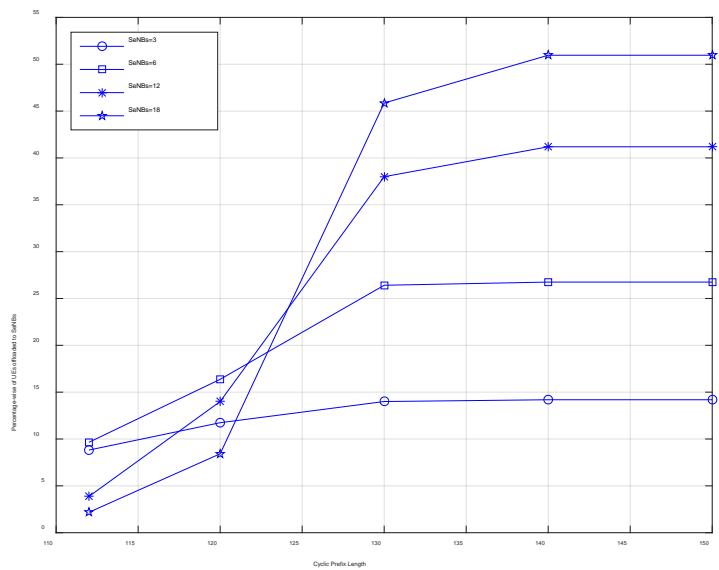

Fig. 8. Percentage-wise of offloaded UEs to SeNBs with the fair association criterion (sec. IV.B).

\section{CONCLUSIONS}

Synchronization in an interference scenario is an important aspect to be considered in OFDM-based dense networks. This work has elucidated the benefits of tuning the transmit precompensation and receive post-compensation with the objective of evaluating the number of TDD SeNBs that can be deployed in a given spatial area when reusing frequency bands.
Moreover, we have demonstrated the necessity of incorporating the signal time offsets with neighboring access points as an additional criterion for user association. We have applied the concept to an FDD scenario where the UL band is partly unused because of the traffic asymmetry. The proposed algorithm allows deploying TDD SeNBs in that unused spectrum and keep carriers orthogonality at its best. The same conclusions can be extended to ultra-dense networks based on TDD SeNBs.

\section{REFERENCES}

[1] DIGITALEUROPE: Call For Timely Harmonisation of the 1452$1492 \mathrm{MHz}$ and $2300-2400 \mathrm{MHz}$ Bands to Support Delivery of EU Radio Spectrum Policy Programme Objectives, Brussels, 21 ${ }^{\text {st }}$ February 2012

[2] N. Bhushan et al., "Network densification: the dominant theme for wireless evolution into 5G,” IEEE Commun. Mag.,vol. 52, pp. 82-89, Feb. 2014.

[3] W. Lei, Z. Mingyu, W. Rongui, "Evolving LTE with Flexible Duplex”, in IEEE Proceedings Globecom 2013

[4] A. Agustin et al., "Efficienct use of paired spectrum bands through TDD small cell deployments”, accepted to IEEE Commun. Mag. Draft version available at https://arxiv.org/abs/1612.02175

[5] 3GPP TR 36.882, "Study on regulatory aspects for flexible duplex for EUTRAN”, Release 13, September 2015

[6] D. Bladsjö, M. Hogan, S. Ruffini, "Synchronization Aspects in LTE Small Cells”, IEEE Comm. Magazine, Sep. 2013

[7] E. Dahlman, S. Parkvall, J. Sköld, 4G LTE/LTE-Advanced for Mobile Broadband, Academic Press, 2011

[8] N. Himayat, et al., "Synchronization Uplink Transmissions from Femto AMS”, IEEE C802.16m-09/3075r2, January 2010

[9] Y. Mostofi, D. C. Cox, "Mathematical Analysis of the Impact of Timing Synchronization Errors on the Performance of an OFDM system", IEEE Trans. on Communications, vol. 54, no. 2, February 2006

[10] K. A. Hamdi, "Precise Interference Analysis of OFDMA Timeasynchronous wireless ad-hoc networks", IEEE Trans. on Wireless Comm., vol. 9, no. 1, Jan. 2010

[11] F. Pantisano, K. Ghaboosi, M. Bennis, M. Latva-Aho, "Interference avoidance via resource scheduling in TDD underlay femtocells", in Proc. IEEE Symposium on Personal Indoor and Mobile Radio Communications (PIMRC), Sep. 2010

[12] 3GPP TR 36.828, "Further enhancements to LTE Time Division Duplex for DL-UL interference management and traffic adaptation”, Release 11, June 2012

[13] Real Wireless. Low-power shared access to spectrum for mobile broadband. Ofcom project MC/073. March 2011

[14] L. Zhao, et al., "An Enhanced Signal-Timing-Offset Compensation Algorithm for Coordianted Multipoint-to-Multiuser Systems”, IEEE Comm. Letters, vol. 18, no. 6, June 2014

[15] S. P. Boyd, L. Vandenberghe, Convex Optimization, Cambridge University Press, 2004

[16] W. Yu, R. Lui, "Dual Methods for Nonconvex Spectrum Optimization of Multicarrier Systems”, IEEE Trans. on Communications, vol. 54, no.7, July 2006

[17] R. Stridh, M. Bengtsson, B. Ottersten, "System Evaluation of Optimal Downlink Beamforming with Congestion Control in Wireless Communication”, IEEE Tran. on Wireless Comm. vol. 5, no. 4, April 06

[18] Y. Wang, K. I. Pedersen, "Performance analysis of enhanced inter-cell interference coordination in LTE-Advanced Heterogeneous Networks", in Proc. IEEE Vehicular Technology Conference (VTC-Spring), 2012 